\title{
Macrocyclic compounds as green corrosion inhibitors for aluminium: electrochemical, surface and quantum chemical studies
}

\author{
K.R. Ansari, ${ }^{1}$ S. Ramkumar, ${ }^{3}$ D.S. Chauhan, ${ }^{1 *}$ Md. Salman, ${ }^{2}$ D. Nalini, ${ }^{3}$ \\ V. Srivastava ${ }^{2}$ and M.A. Quraishi ${ }^{1^{*}}$ \\ ${ }^{1}$ Center of Research Excellence in Corrosion, Research Institute, King Fahd University \\ of Petroleum and Minerals, Dhahran 31261, Saudi Arabia \\ ${ }^{2}$ Department of Chemistry, Indian Institute of Technology (Banaras HinduUniversity), \\ Varanasi 221005, India \\ ${ }^{3}$ Department of Chemistry, PSGR Krishnammal College for Women, Coimbatore 641004, \\ Tamil Nadu, India \\ *E-mail: dschauhan.bhu@gmail.com,maquraishi.apc@itbhu.ac.in
}

\section{Abstract}

In the present work, two macrocyclic compounds namely 5,6,11,12-tetramethyl1,2,4,7,8,10-hexaazacyclododeca-4,6,10,12-tetraene-3,9-dithione (TMCD) and 5,6,11,12tetraphenyl-1,2,4,7,8,10-hexaazacyclododeca-4,6,10,12-tetraene-3,9-dithione

(TMPD) have been synthesized and for the first time reported as inhibitors against the corrosion of aluminium in $1 \mathrm{M}$ hydrochloric acid. The synthesized compounds were characterized using ${ }^{1} \mathrm{H}$ NMR and ${ }^{13} \mathrm{C}$ NMR. The corrosion inhibition behaviour of TMCD and TMPD was studied using gravimetric measurements, electrochemical impedance spectroscopy and potentiodynamic polarization studies. The surface analysis was carried out using AFM and SEM. Theoretical studies on the adsorption behaviour of inhibitor were carried out using the Density Functional Theory method. A detailed study of the effect of temperature and the influence of immersion time is presented using weight loss technique. Potentiodynamic polarization study proves that the corrosion inhibition efficiencies of TMCD and TMPD at $400 \mathrm{mg} \mathrm{L}^{-1}$ concentration are $97.30 \%$ and $89.71 \%$ respectively. Both the inhibitors behaved as mixed type but exhibit cathodic predominance. The results of EIS-Nyquist plots show inductive behavior at low frequency characteristic of the electrochemical behaviour of aluminium. The increase in polarization resistance with concentration suggests the adsorption and corrosion inhibition behaviour for both TMCD and TMPD. The AFM studies reveal a considerable decrease in surface roughness in the presence of inhibitors compared to blank sample and the SEM images show a highly smooth surface of the metal sample in the presence of inhibitors. The Fukui functions, global softness and Mulliken charges reveal that the sites for electrophilic attack are the hetero atoms. The frontier molecular orbital energies, the molecular orbital energy gap and the other quantum 
chemical parameters corroborated the experimental observations and suggested a better performance of TMCD compared to TMPD molecule.

Received: June 25, 2018. Published: September 28, 2018

doi: $\underline{10.17675 / 2305-6894-2018-7-3-13}$

Keywords: aluminium, corrosion inhibition, adsorption, potentiodynamic polarization, electrochemical impedance, scanning electron microscopy.

\section{Introduction}

Corrosion of aluminium especially its alloys has been a subject of numerous studies due to their high technological value and wide range of industrial applications especially in aerospace and household industries. Aluminium relies on the formation of a compact, adherent passive oxide film for its corrosion immunity in various environments. This surface film is amphoteric and dissolves substantially when the metal is exposed to high concentrations of acids and bases [1,2].

$\mathrm{HCl}$ is usually used in industry for acid cleaning, chemical or electrochemical etching and acid pickling of aluminium, but at the same time it causes its corrosion. Therefore, in order to prevent its dissolution in $\mathrm{HCl}$, corrosion inhibitors are used [3-6]. These inhibitor molecules show their inhibition property by getting adsorbed on the aluminium surface, either physically or chemically through heteroatoms $\mathrm{N}, \mathrm{O}, \mathrm{S}$ and $\mathrm{P}$, conjugate double bonds or $\pi$ electrons of aromatic rings.

Most of the organic inhibitors are toxic in nature [7-12]. Therefore their use is not safe. In view of environmental safety, at present, research has been focused on the development of ecofriendly corrosion inhibitors, in order to protect environment from hazardous effect of toxic inhibitors. Keeping this view in mind, two macrocyclic compounds have been synthesized and evaluated as corrosion inhibitors for aluminium in $\mathrm{HCl}$ solution. These compounds have been choosen as inhibitors because of there biological activities [13] and because of their fascinating molecular structure, the presence of $\pi$ electrons or non bonding electrons. In addition to these structural features, planarity of these molecules further facilitates the formation of a strong bond between metal and macrocyclic molecules.

Recently quantum chemical studies have become an important tool for correlating the molecular structure with there inhibition properties [14].

The present paper reports the inhibition action of two macrocyclic compounds, namely 5,6,11,12-tetramethyl-1,2,4,7,8,10-hexaazacyclododeca-4,6,10,12-tetraene-3,9dithione (TMCD) and 5,6,11,12-tetraphenyl-1,2,4,7,8,10-hexaazacyclododeca-4,6,10,12tetraene-3,9-dithione (TMPD) on aluminium using weight loss, electrochemical impedance spectroscopy (EIS), Tafel polarization, SEM, AFM and quantum chemical calculations. 


\section{Experimental}

\subsection{Materials}

The test solution i.e. $1 \mathrm{M} \mathrm{HCl}$ was prepared by diluting $37 \%$ analytical grade $\mathrm{HCl}$ with double distilled water. The composition of aluminium sample used is as follows: $\mathrm{Si}=0.77$, $\mathrm{Fe}=0.93, \mathrm{Cu}=0.02, \mathrm{Mn}=0.11, \mathrm{Mg}=0.01, \mathrm{Zn}=0.01, \mathrm{Cr}=0.05, \mathrm{Ti}=0.02, \mathrm{~V}=0.01$, $\mathrm{Ga}=0.01$ and balance Al. Aluminium strips with an exposed area of $1 \mathrm{~cm}^{2}$ were used in electrochemical experiments, while for weight loss the dimensions of aluminium were $2.0 \mathrm{~cm} \times 2.5 \mathrm{~cm} \times 0.2 \mathrm{~cm}$.

\subsection{Synthesis of inhibitors}

The investigated organic compounds were synthesized in the laboratory by the reported procedure [15]. In a round bottom flask diacetyl or benzil was taken and mixed with thiosemicarbazide (molar ratio of 1:2) in the presence of $1 \mathrm{ml} \mathrm{HCl}$ and ethanol. The mixture was subjected to reflux for 4-6 h and the resulting solution was kept overnight. The product thus obtained was filtered and recrystallized using ethanol. The scheme of synthesis is shown in Figure 1. The molecular structure, abbreviation and spectral data are tabulated in Table 1.

\subsection{Electrochemical measurements}

Electrochemical measurements were performed using a three electrode cell assembly, in which aluminium strip was used as working electrode, platinum as counter and saturated calomel as reference electrodes. All measurements were carried out using a Gamry Potentiostat/Galvanostat (Model G-300) at $308 \mathrm{~K}$ temperature and data analysis was carried out by using Echem Analyst 5.0 software package.

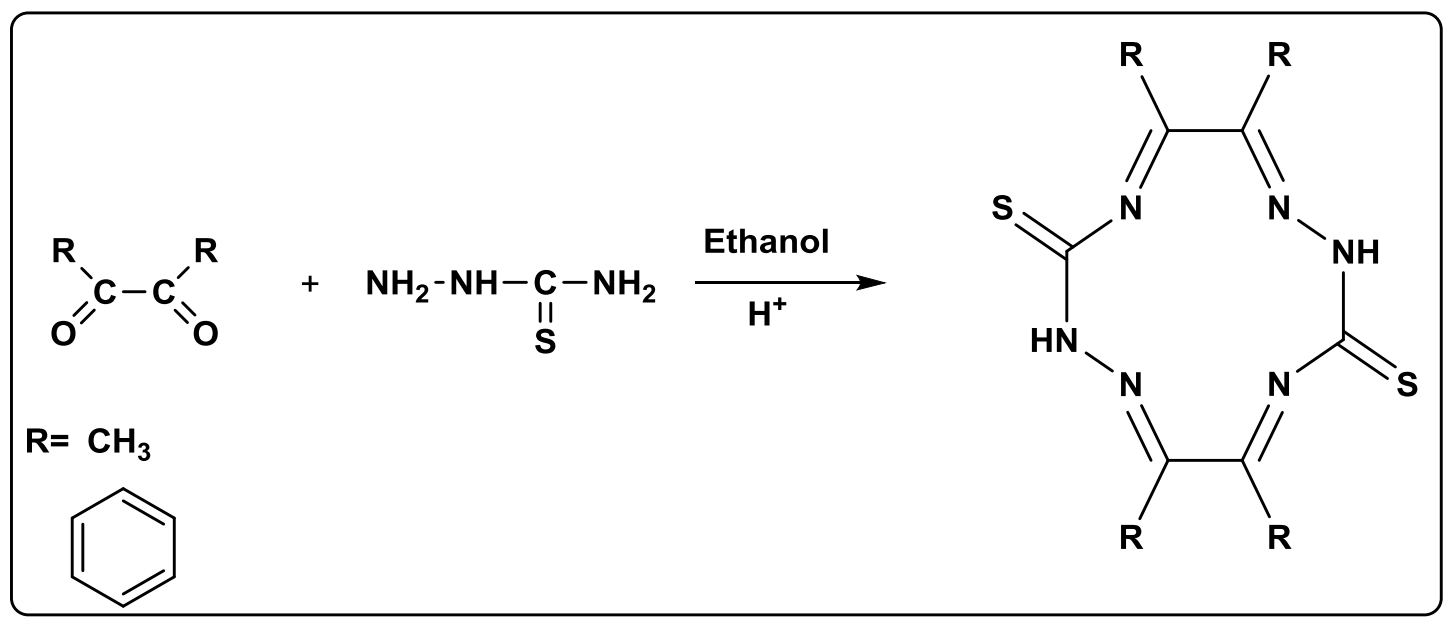

Figure 1. Synthetic route of inhibitors. 
Table 1. Molecular structure of inhibitors.

Inhibitor
$\begin{gathered}\text { 5,6,11,12-tetramethyl- } \\ \text { 1,2,4,7,8,10- } \\ \text { tetraene-3,9-dithione(TMCD) }\end{gathered}$
$\begin{gathered}\text { 5,6,11,12-tetraphenyl- } \\ \text { 1,2,4,7,8,10- } \\ \text { tetraene-3,9-dithione (TMPD) }\end{gathered}$

The potentiodynamic polarization measurements were performed by changing the electrode potential automatically from -250 to $+250 \mathrm{mV}$ versus OCP at a scan rate of $1 \mathrm{mV} \mathrm{s}^{-1}$.

EIS measurements were carried out using AC signal of amplitude $10 \mathrm{mV}$ peak to peak in the frequency range of $100 \mathrm{kHz}$ to $0.01 \mathrm{~Hz}$ at open circuit potential (OCP). All electrochemical experiments were done after immersing the aluminium sample (working electrode) for $30 \mathrm{~min}$ in $1 \mathrm{M} \mathrm{HCl}$ in the absence and presence of inhibitors.

\subsection{Surface morphology}

Surface analysis was carried out by using SEM and AFM, in order to find out the morphological changes occurring on the aluminium surface in the absence and presence of inhibitors. In both SEM and AFM, aluminium specimens were first exposed to $1 \mathrm{M} \mathrm{HCl}$ solution in the absence and presence of optimum concentration $(400 \mathrm{mg} / \mathrm{L})$ of TMCD and TMPD for $3 \mathrm{~h}$ at $308 \mathrm{~K}$ respectively. The SEM was performed by a Ziess SUPRA 40 instrument model, with 5kx magnification and the instrument used for AFM is (AFM, NTMDT SOLVER Next AFM/STM). The scan size of sample for AFM is $10 \mu \mathrm{m} \times 10 \mu \mathrm{m}$. 


\subsection{Quantum chemical study}

The inhibitor compounds were fully optimized using B3LYP Density Functional Theory formalism (DFT) with 6-31 G (d, p) basis set using Gaussian-03 [16]. The following quantum chemical parameters were calculated: Energy of the Highest Occupied Molecular Orbital $\left(E_{\mathrm{HOMO}}\right)$, Energy of the Lowest Unoccupied Molecular Orbital ( $\left.E_{\mathrm{LUMO}}\right)$, Energy gap between HOMO and LUMO $(\Delta E)$, dipole moment $(\mu)$, ionization potential (IP), electron affinity (EA), Global hardness $(\eta)$, absolute softness $(\sigma)$, electronegativity $(\chi)$, fraction of electron transferred $(\Delta N)$, Mulliken charge density and Fukui function $(f)$.

\section{Results and discussion}

\subsection{Weight loss measurement}

\subsubsection{Effect of temperature}

Weight loss measurements were carried out in the temperatures range of $308-338 \mathrm{~K}$ in absence and presence of optimum concentration $\left(400 \mathrm{mg} \mathrm{L}^{-1}\right)$ of inhibitors. The results obtained are shown in Figure 2(a). Figure reveals that the inhibition efficiency decreases with increase in temperature, which is due to the desorption of inhibitor molecules from the metal surface.

The temperature dependence of corrosion rate is expressed by Arrhenius equation:

$$
\log C_{\mathrm{R}}=\frac{-E_{\mathrm{a}}}{2.303 R T}+\log \lambda
$$

where, $E_{\mathrm{a}}$ represents the apparent activation energy and $\lambda$ denotes the pre-exponential factor. The activation energy was calculated by plotting a graph between $\log C_{\mathrm{R}}$ and $1 / T$ and is represented in Figure 2(b). From the slope of the obtained straight line, the activation energy was calculated and presented in Table 2 . An inspection of Table 2 reveals that the values of activation energy are higher in the presence of inhibitors than in their absence. This indicates that as the temperature increased, there is an appreciable decrease in the adsorption of inhibitors, which in turn increases the corrosion rate because more metal surface area is now exposed to the acidic environment.

Transition state theory was used for calculating standard enthalpy and entropy of activation respectively and is given as follows:

$$
C_{\mathrm{R}}=\frac{R T}{N h} \exp \left\{\frac{\Delta S_{\mathrm{a}}^{0}}{R}\right\} \exp \left\{-\frac{\Delta H_{\mathrm{a}}^{0}}{R T}\right\}
$$

where $h$ is Plank's constant, $N$ is Avogadro number, $\Delta H_{\mathrm{a}}^{0}$ and $\Delta S_{\mathrm{a}}^{0}$ are the standard enthalpy and standard entropy of activation, respectively. Plots of $\log \left(C_{\mathrm{R}} / T\right)$ versus $1 / T$ (Figure 2c) gave straight lines with $\left(-\Delta H_{\mathrm{a}}^{0} / 2.303 R\right)$ as the slope values and intercepts equal to $\left[\log (R / N h)+\left(-\Delta S_{\mathrm{a}}^{0}\right) / 2.303 R\right]$. The values of above parameters are depicted in 
Table 2. The values of $\Delta H_{\mathrm{a}}^{0}$ and $E_{\mathrm{a}}$ are close to each other as expected from the concept of transition state theory. Inspection of Table 2 reveals that the value of $\Delta H_{\mathrm{a}}^{0}$ increased in the presence of the inhibitors than in their absence, which indicates higher protection from corrosion by increasing the energy barrier for the corrosion reaction. Also, after comparing the values of $\Delta S_{\mathrm{a}}^{0}$, it is clear that $\Delta S_{\mathrm{a}}^{0}$ values are increased in the presence of the inhibitors as compared to that in their absence. In absence of inhibitors the rate determining step in the transition state are having more ordered arrangement as compared to the initial state and thus a high value is obtained for the entropy of activation. However in the presence of inhibitors, the rate determining step, which is the discharge of hydrogen ions to form adsorbed hydrogen atoms is retarded because the metal surface is covered by the inhibitor molecules, this will make the system in a random arrangement, and this causes the increase in entropy of activation.
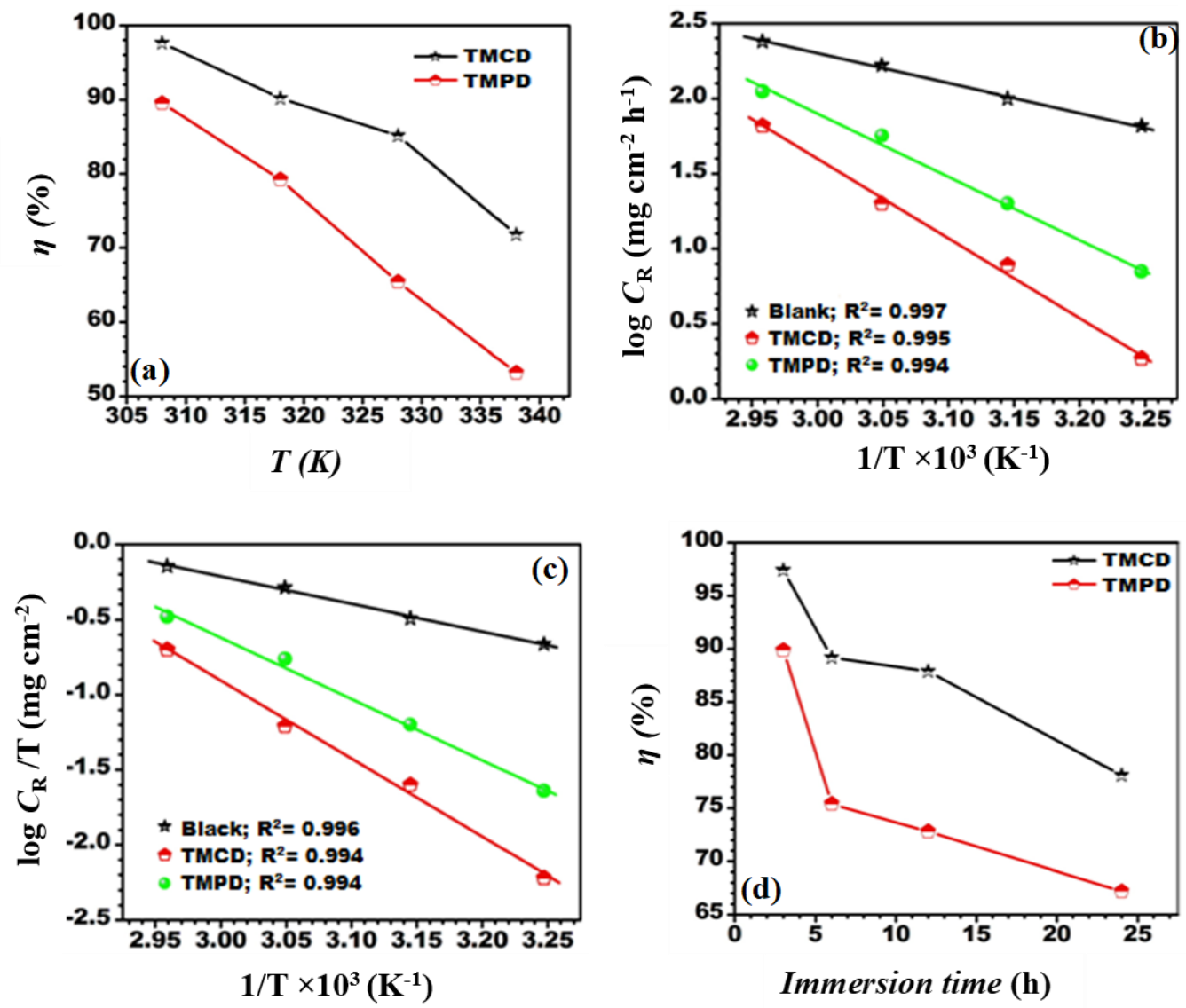

Figure 2. (a) Variation of inhibition efficiency $(\eta \%)$ with solution temperature $(308-338 \mathrm{~K})$ at optimum concentration of inhibitors; (b) Arrhenius plots of the corrosion rate $\left(C_{\mathrm{R}}\right)$ of mild steel in $1 \mathrm{M} \mathrm{HCl}$ in the absence and presence of optimum concentration of inhibitors; (c) Transition-state plots of the corrosion rate $\left(C_{\mathrm{R}}\right)$ of mild steel in $1 \mathrm{M} \mathrm{HCl}$ in absence and presence of optimum concentration of inhibitors; and (d) Variation of inhibition efficiency $(\eta \%)$ with immersion time (h) at optimum concentration of inhibitors. 
Table 2. Thermodynamic parameters for the adsorption of inhibitor on aluminium in $1 \mathrm{M} \mathrm{HCl}$ at optimum concentration of inhibitors.

\begin{tabular}{|c|c|c|c|}
\hline Inhibitor & $E_{\mathrm{a}}\left(\mathrm{kJ} \mathrm{mol}^{-1}\right)$ & $\Delta H_{\mathrm{a}}^{0}\left(\mathrm{~kJ} \mathrm{~mol}^{-1}\right)$ & $\Delta S_{\mathrm{a}}^{0}\left(\mathrm{~J} \mathrm{~K}^{-1} \mathrm{~mol}^{-1}\right)$ \\
\hline Blank & 37.76 & 35.06 & -96.49 \\
\hline TMCD & 100.75 & 98.78 & 81.20 \\
\hline TMPD & 80.59 & 78.18 & 25.18 \\
\hline
\end{tabular}

\subsubsection{Effect of immersion time}

Aluminium samples were immersed for $24 \mathrm{~h}$ in absence and presence of optimum concentration (400 $\mathrm{mg} \mathrm{L}^{-1}$ ) of inhibitors and are shown in Figure 2(d). It is clear from Figure 2(d) that the increase in the immersion time has very pronounced effect on the inhibition efficiency and the inhibition efficiency decreases slowly for TMCD from 3-6 $\mathrm{h}$ but in case of TMPD this decrease is more. But after $6 \mathrm{~h}$ the trend of decreae in inhibition efficiency is almost same in both the inhibitors.
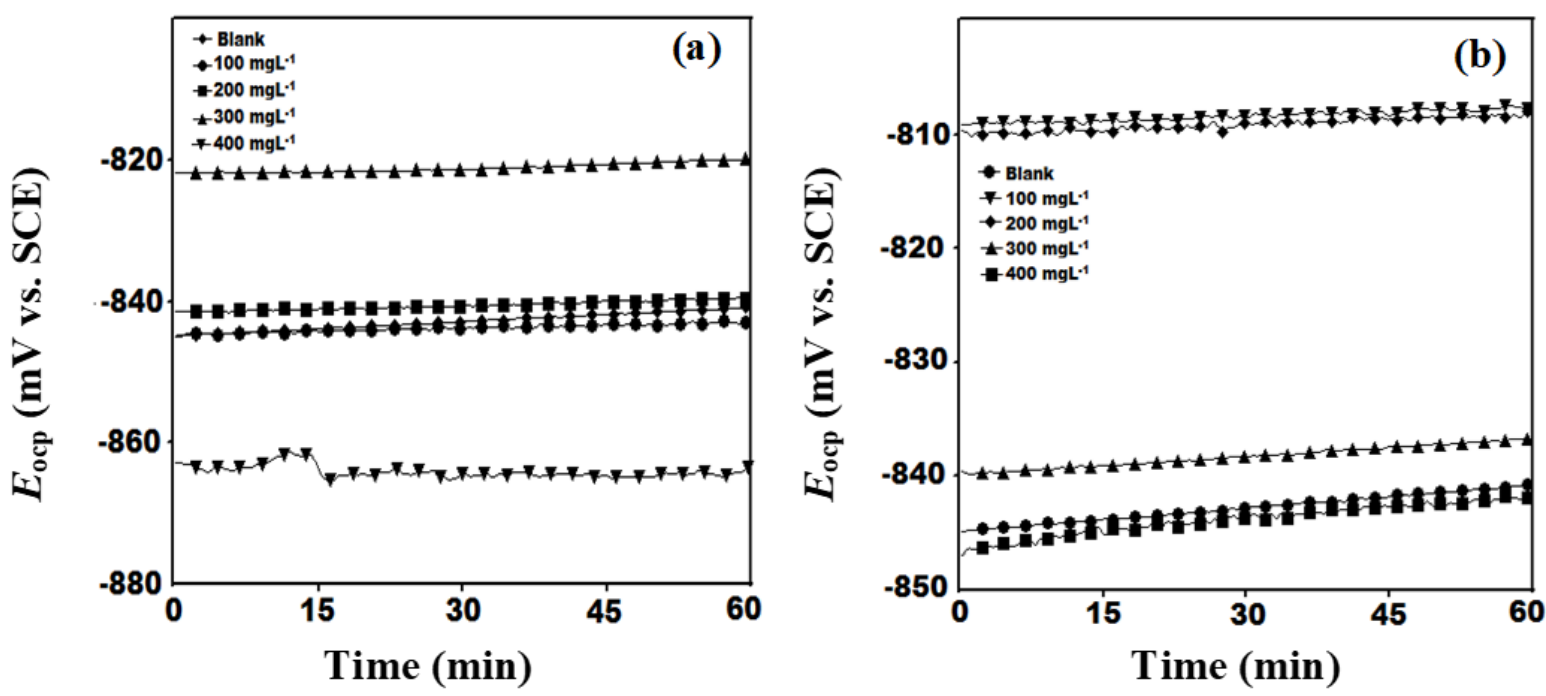

Figure 3. OCP curve (a) TMCD (b) TMPD.

\subsection{Electrochemical measurements}

\subsubsection{Open Circuit Potential Measurements}

The time dependency of open circuit potential (OCP) in absence and presence of inhibitors was recorded for 60 minutes and is shown in Figure 3(a,b). The observation of figure reveals that as the time passes the potential curves in presence of inhibitors moves towards both negative and positive direction with respect to blank. 


\subsubsection{Potentiodynamic polarization}

The potentiodynamic polarization curves at $308 \mathrm{~K}$ in $1 \mathrm{M} \mathrm{HCl}$ are given in Figure 4(a,b). Both the inhibitors (TMCD and TMPD) cause the decrease of corrosion process i.e. shifting both the cathodic and anodic curves towards lower current density. Also there is no definite trend for $E_{\text {corr }}$ values i.e. shifting both towards cathodic and anodic side. This reveals that these inhibitors are acting as mixed type. After observing Figure 4(a,b), it can be observed that cathodic curves are having parallel Tafel lines, which indicates that evolution of hydrogen is under the activation controlled reaction. But linear Tafel regions for anodic curves are difficult to recognize. So, the corrosion current density $\left(i_{\text {corr }}\right)$ values are obtained by extrapolating the linear region of cathodic curves upto the corrosion potential [17].
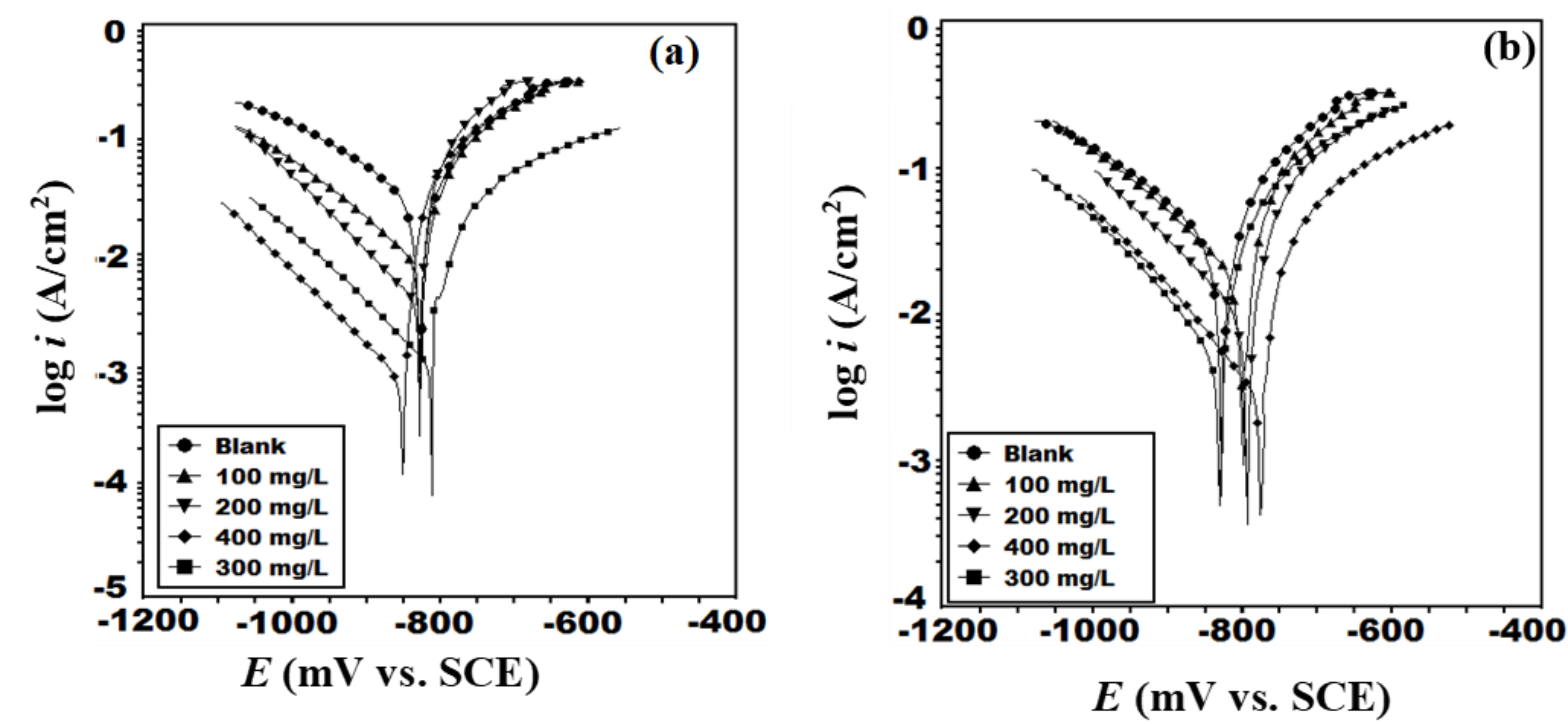

Figure 4. Tafel curves for aluminium in $1 \mathrm{M} \mathrm{HCl}$ in absence and presence of different concentrations of inhibitors at $308 \mathrm{~K}$ (a) TMCD (b) TMPD.

The electrochemical parameters including corrosion potential $\left(E_{\text {corr }}\right)$, corrosion current density $\left(i_{\text {corr }}\right)$, inhibition efficiency $(\eta \%)$ and cathodic Tafel slope $\left(\beta_{\mathrm{c}}\right)$ are given in Table 3.

The values of $\eta \%$ were calculated by using the following equation [18-20]:

$$
\eta \%=\left(1-\frac{I_{\text {corr(i) }}}{I_{\text {corr }}}\right) \times 100
$$

From the table, it can be observed that $I_{\text {corr }}$ values in the presence of the inhibitors were lower than in their absence [21,22] and also the $I_{\text {corr }}$ values decrease as the concentration of the inhibitors increases. The values of cathodic Tafel slope $\left(\beta_{\mathrm{c}}\right)$ changes significantly with the addition of different concentration of TMCD and TMPD, which means that inhibitors are adsorbing dominantly on cathodic sites and resulting the 
inhibition of cathodic reactions. So, overall discussion reveals that both the inhibitors are mixed type but affecting more the cathodic reaction [23].

Table 3. Tafel polarization parameters for the corrosion of aluminium in $1 \mathrm{M} \mathrm{HCl}$ solution containing different concentrations of inhibitors at $308 \mathrm{~K}$ (immersion time $30 \mathrm{~min}$ ).

\begin{tabular}{|c|c|c|c|c|c|}
\hline Inhibitor & $\begin{array}{l}\text { Conc. } \\
\text { (mg/L) }\end{array}$ & $\begin{array}{c}\boldsymbol{E}_{\text {corr }} \\
(\mathbf{m V} / \mathbf{S C E})\end{array}$ & $\begin{array}{c}i_{\text {corr }} \\
\left(\mu \mathrm{A} / \mathbf{c m}^{2}\right)\end{array}$ & $\begin{array}{c}-\boldsymbol{\beta}_{\mathbf{c}} \\
(\mathbf{m V} / \mathbf{d e c})\end{array}$ & $\stackrel{\boldsymbol{\eta}}{(\%)}$ \\
\hline Blank & - & -828.6 & 29790 & 247.9 & - \\
\hline \multirow{4}{*}{ TMCD } & 100 & -826.6 & 9479 & 206.0 & 68.18 \\
\hline & 200 & -828.7 & 3718 & 153.3 & 87.51 \\
\hline & 300 & -811.0 & 1114 & 165.6 & 96.26 \\
\hline & 400 & -851.0 & 802.4 & 156.7 & 97.30 \\
\hline \multirow{4}{*}{ TMPD } & 100 & -799.9 & 14140 & 224.0 & 52.53 \\
\hline & 200 & -796.9 & 9878 & 200.0 & 66.84 \\
\hline & 300 & -830.8 & 5556 & 183.5 & 81.34 \\
\hline & 400 & -776.1 & 3063 & 176.3 & 89.71 \\
\hline
\end{tabular}

\subsubsection{Electrochemical impedance spectroscopy}

Nyquist plots in absence and presence of different concentrations of inhibitors at $308 \mathrm{~K}$ are shown in Figure 5(a,b). It could easily be observed that there is a large capacitive loop existing at higher frequencies (HF) followed by a large inductive loop at low frequency (LF). Survey of literature reveals that [24], corrosion of aluminium in $\mathrm{HCl}$ always consists of a small inductive loop at LF as compared to capacitive loop. However, in the present study, the size of inductive loop is almost equal to that of capacitive loop, making whole diagram with an elliptical shape [25]. Also the shape of Nyquist curves in the absence and presence of inhibitors are similar at all concentrations, which reveals that corrosion mechanism process remains unchanged [25].

The origin of the large inductive loop at low frequency is still unclear. Adsorbed charged intermediates may result in an inductive loop [26]. This is more pronounced when the intermediates are strongly adsorbed. Lenderink et al. [27] have attributed to the relaxation of adsorbed species like $H_{\text {ads }}^{+}$. Other suggested relaxation adsorbed intermediates include $\mathrm{Cl}^{-}$[28], oxygen ion [29] on the electrode surface. It might be also attributed to the re-dissolution of the oxide layer surface or Al dissolution [28] at low frequencies.

The equivalent circuit used to extract the data is shown in Figure 5(c). This circuit consists of $R_{\mathrm{s}}$ (solution resistance), $R_{\mathrm{t}}$ (charge transfer resistance), CPE (constant phase element), $R_{\mathrm{L}}$ (inductive resistance) and $L$ (inductance) respectively. The polarization resistance $\left(R_{\mathrm{p}}\right)$ can be calculated from the following equation [30]: 


$$
R_{\mathrm{P}}=\frac{R_{\mathrm{t}} R_{\mathrm{L}}}{R_{\mathrm{t}}+R_{\mathrm{L}}}
$$

Then the inhibition efficiency $(\eta \%)$ is calculated from $R_{\mathrm{p}}$ using the following relation:

$$
\eta \%=\frac{R_{\mathrm{P}(\mathrm{inh})}-R_{\mathrm{P}(0)}}{R_{\mathrm{P}(\mathrm{inh})}}
$$

where $R_{\mathrm{p}(0)}$ and $R_{\mathrm{p} \text { (inh) }}$ are polarization resistance in the absence and presence of inhibitor, respectively.
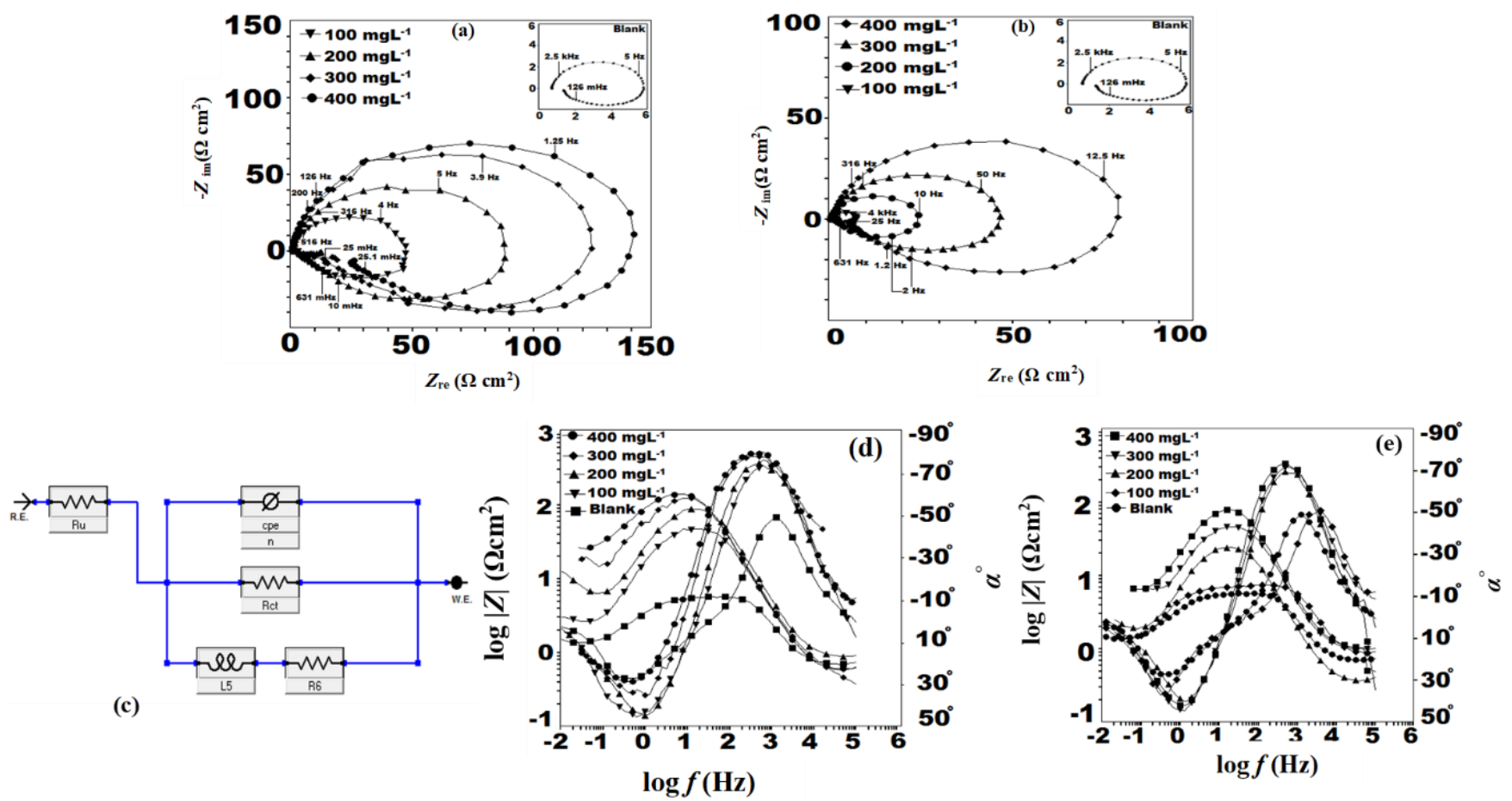

Figure 5. Nyquist plots for aluminium in $1 \mathrm{M} \mathrm{HCl}$ in absence and presence of different concentration of inhibitors at $308 \mathrm{~K}$ (a) TMCD (b) TMPD; (c) Equivalent circuit model used to fit the EIS data; (d) Bode plots in $1 \mathrm{M} \mathrm{HCl}$ in absence and presence of different concentration of inhibitors at $308 \mathrm{~K}$; (d) TMCD; and (e) TMPD.

The calculated impedance parameters are listed in Table 4. The precision of the fitted data was evaluated by chi-squared $\left(\chi^{2}\right)$. The values of $\chi^{2}$ are very small (Table 4 ), which supports that the equivalent circuit is ideal for fitting. Also the values of $n$ in presence of inhibitors are greater i.e. approaching towards 1 than in their absence, which reveals that the surface becomes regular in presence of inhibitors. The $R_{\mathrm{S}}$ values are very small, which confirms that the IR drop is small in the present experiments. The increasing values of both $R_{\mathrm{t}}$ and $R_{\mathrm{p}}$ with the addition of inhibitors reveals that the corrosion process in presence of inhibitors becomes slower. In presence of inhibitors the values of $C P E$ are smaller than in the absence which arises by decrease in the local dielectric constant and/or an increase in the thickness of the electrical double layer, suggesting that the action of inhibitor occurs by 
adsorption at the aluminium/solution interface [31]. Table 3 reveals that inhibition efficiency increases as the concentration of inhibitors increases, and follows the order: TMCD > TMPD.

Bode impedence and phase angle plots are given in Figure 5(d,e). These Bode plots consist of two time constants, namely, a medium frequency time constant, which is corresponding to the capacitive behaviour of the aluminium surface, and a low frequency time constant releated to the inductive behaviour, which is accompainied with the decrease of the impedence and a positive phase shift with the lowering of the frequency, corresponding to the relaxation process of adsorbed species in the oxide flim covering the aluminium surface [26] or re-dissolution of the oxide layer surface [32]. Also the value of phase angle and Bode impedence values show an increasing trend with increase in the inhibitors concentration, which revelas that the inhibitors are adsorbed on the aluminium surface and check the corrosion process.

Table 4. EIS parameters for the corrosion of aluminium in $1 \mathrm{M} \mathrm{HCl}$ solution containing different concentrations of inhibitors at $308 \mathrm{~K}$ (immersion time $30 \mathrm{~min}$ ).

\begin{tabular}{|c|c|c|c|c|c|c|c|c|c|}
\hline Inhibitor & $\begin{array}{l}\text { Conc. } \\
\mathrm{mg} / \mathrm{L}\end{array}$ & $n$ & $\begin{array}{c}R_{\mathrm{t}} \\
\left(\Omega \mathrm{cm}^{2}\right)\end{array}$ & $\begin{array}{c}R_{\mathrm{L}} \\
\left(\Omega \mathrm{cm}^{2}\right)\end{array}$ & $\begin{array}{c}L \\
\left(\mathbf{H ~ c m}^{2}\right)\end{array}$ & $\begin{array}{c}R_{\mathrm{P}} \\
\left(\Omega \mathrm{cm}^{2}\right)\end{array}$ & $\begin{array}{c}\mathrm{CPE} \\
\left(\mu \mathrm{F} \mathrm{cm} \mathrm{cm}^{-2}\right)\end{array}$ & $\begin{array}{c}H \\
(\%)\end{array}$ & $\chi^{2}$ \\
\hline Blank & - & 0.780 & 5.1 & 0.8 & 0.756 & 0.691 & 157.7 & - & $0.78 \times 10^{-2}$ \\
\hline \multirow{4}{*}{ TMCD } & 100 & 0.965 & 47.16 & 2.350 & 2.884 & 2.238 & 52.21 & 69.12 & $1.3 \times 10^{-2}$ \\
\hline & 200 & 0.982 & 90.37 & 6.019 & 4.452 & 5.643 & 45.13 & 87.75 & $1.8 \times 10^{-2}$ \\
\hline & 300 & 0.986 & 130.4 & 11.58 & 12.51 & 10.635 & 94.62 & 93.50 & $9.2 \times 10^{-2}$ \\
\hline & 400 & 0.988 & 146.2 & 32.53 & 27.68 & 26.609 & 53.42 & 97.40 & $0.87 \times 10^{-2}$ \\
\hline \multirow{4}{*}{ TMPD } & 100 & 0.798 & 8.90 & 0.9 & 1.02 & 0.817 & 87.01 & 15.42 & $0.69 \times 10^{-2}$ \\
\hline & 200 & 0.950 & 24.50 & 1.848 & 1.227 & 1.717 & 129.5 & 59.77 & $1.4 \times 10^{-2}$ \\
\hline & 300 & 0.960 & 47.20 & 5.700 & 1.807 & 5.085 & 52.64 & 86.41 & $2.6 \times 10^{-2}$ \\
\hline & 400 & 0.977 & 80.72 & 7.554 & 3.114 & 6.907 & 52.43 & 89.99 & $5.4 \times 10^{-2}$ \\
\hline
\end{tabular}

\subsection{Surface characterization: SEM and AFM}

SEM images are shown in Figure 6(a-c). In the absence of inhibitors aluminium surface was severely damaged (Figure 6a), whereas, in the presence of TMCD and TMPD (Figure $3(\mathrm{~b}, \mathrm{c}))$, the corrosion was tangibly suppressed. This nearly smooth morphology of aluminium surface reveals that the adsorption of inhibitors has occurred, which is acting as a barrier between aluminium and the corrosive medium.

The three dimensional (3D) AFM morphologies in the absence and presence of inhibitors are given in Figure 7(a-c). The parameters calculated are $R_{\mathrm{q}}$ (Root-mean-square roughness), $R_{\mathrm{a}}$ (average roughness), $R_{\mathrm{z}}$ (average of roughness values taken from ten points) and $R_{\Delta \mathrm{q}}$ (Root mean square slope of roughness). 
Figure 7(a) represents a highly corroded surface in absence of inhibitors but as the inhibitor is added the surface morphology becomes smooth (Figure 7(b,c)), suggesting inhibitor film formation over the aluminium surface. Also from Table 5, it could be observed that the values of $R_{\mathrm{q}}, R_{\mathrm{a}}, R_{\mathrm{z}}$ are large in the absence of inhibitor, revealing a greater surface roughness. In presence of inhibitors the values of all the calculated parameters are reduced and this reduction in TMCD is larger than TMPD, which confirms that the surface becomes smoother and this smoothness occurs due to the formation of compact protective film of inhibitors (TMCD and TMPD).

The last parameter $R_{\Delta \mathrm{q}}$ represents the corrosion resistance behavior of the material. Table 5 revealed that in the absence of inhibitor, $R_{\Delta q}$ value is less as compared to that in the presence of inhibitors. These data justify that in absence of inhibitor the aluminium is going to corrode at the greater extent than in presence of inhibitors. This reduction in corrosion in presence of inhibitors further supports the formation of a protective film of inhibitor molecules.
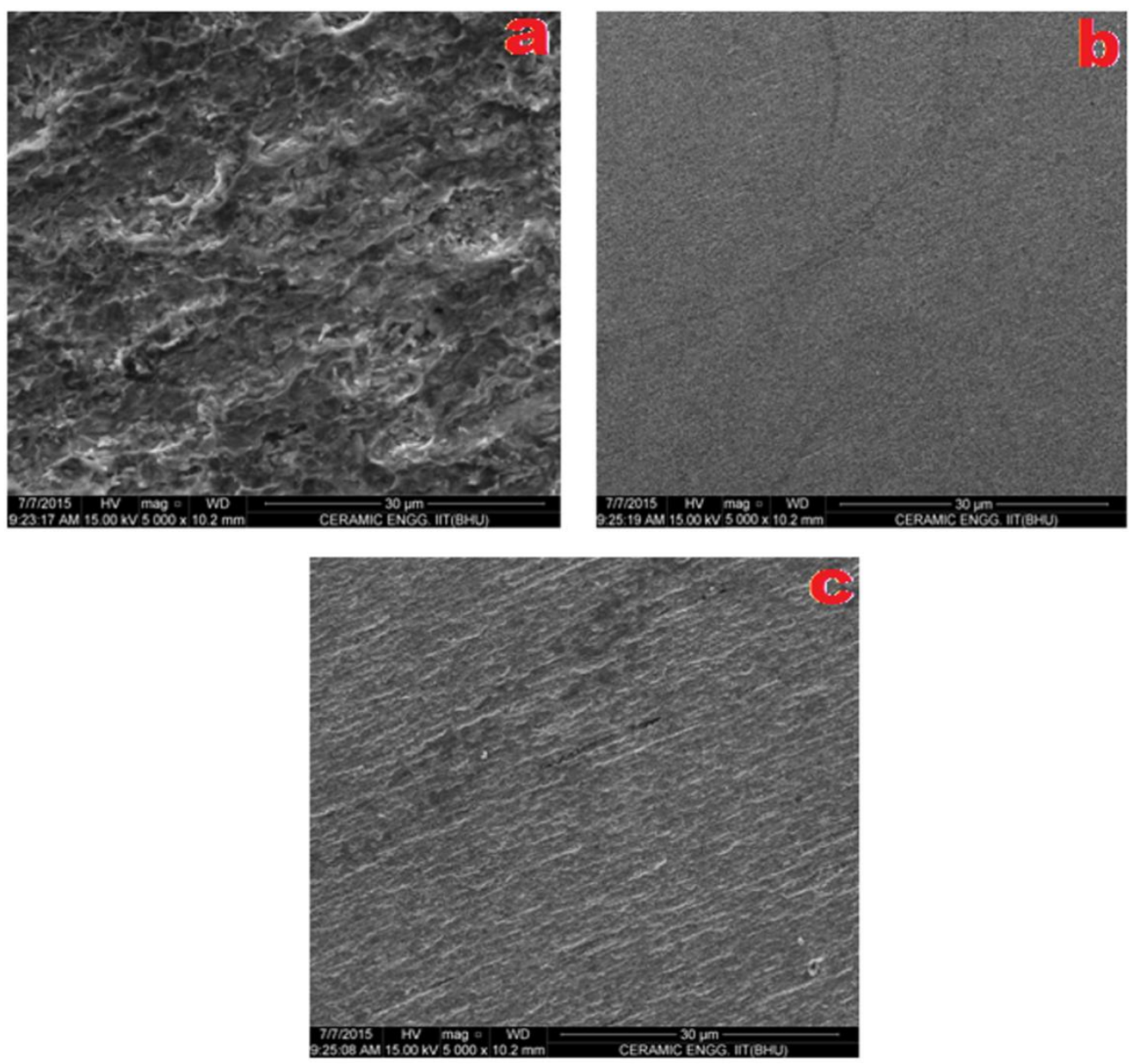

Figure 6. SEM images (a) unhibited (b) TMCD (c) TMPD. 

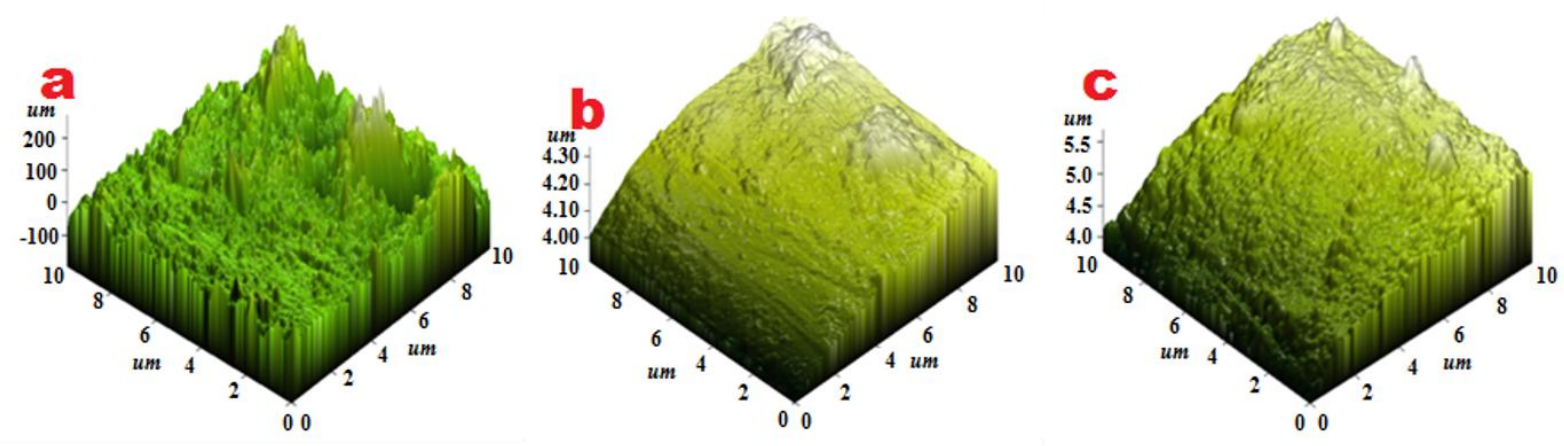

Figure 7. AFM images (a) unhibited (b) TMCD (c) TMPD.

Table 5. Roughness parameters obtained from AFM.

\begin{tabular}{ccccc}
\hline Sample & $\boldsymbol{R}_{\mathbf{q}}(\boldsymbol{\mu} \mathbf{m})$ & $\boldsymbol{R}_{\mathbf{a}}(\boldsymbol{\mu} \mathbf{m})$ & $\boldsymbol{R}_{\Delta \mathbf{q}}(\boldsymbol{\mu m})$ & $\boldsymbol{R}_{\mathbf{z}}$ \\
\hline Blank & 20.440 & 16.102 & 0.112 & 62.175 \\
TMCD & 0.022 & 0.016 & 0.162 & 0.062 \\
TMPD & 6.517 & 5.351 & 0.151 & 16.593 \\
\hline
\end{tabular}

\subsection{Quantum chemical studies using density functional theory (DFT)}

The optimized geometry and the Frontier orbital structure of the inhibitor molecules are presented in Figures 8 and 9. The calculated quantum chemical parameters necessary for discussion of the reactivity of the selected inhibitors are reported in Table 6.

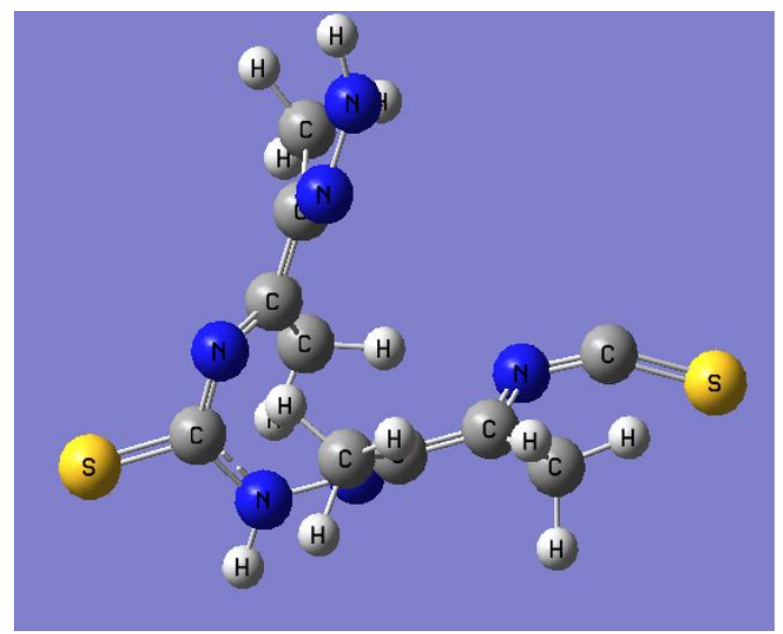

TMCD

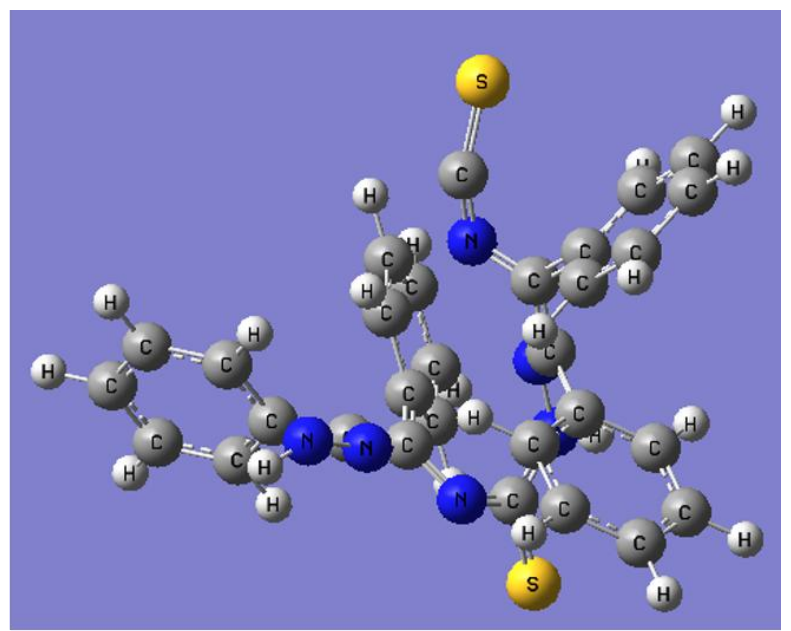

TMPD

Figure 8. Optimized structures of inhibitors. 


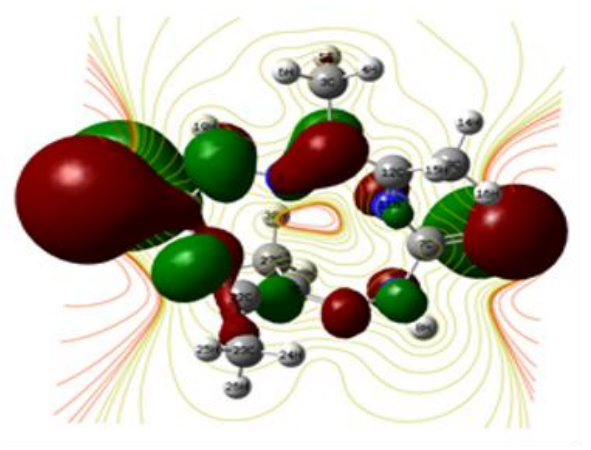

HOMO

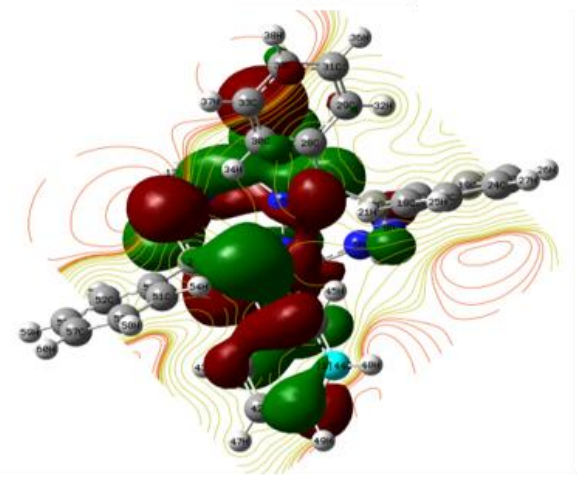

HOMO (a)

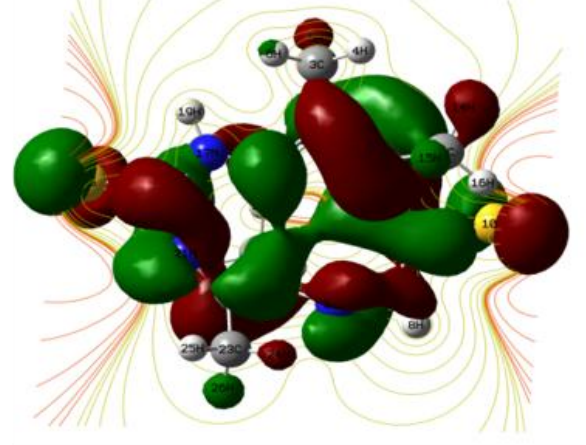

LUMO

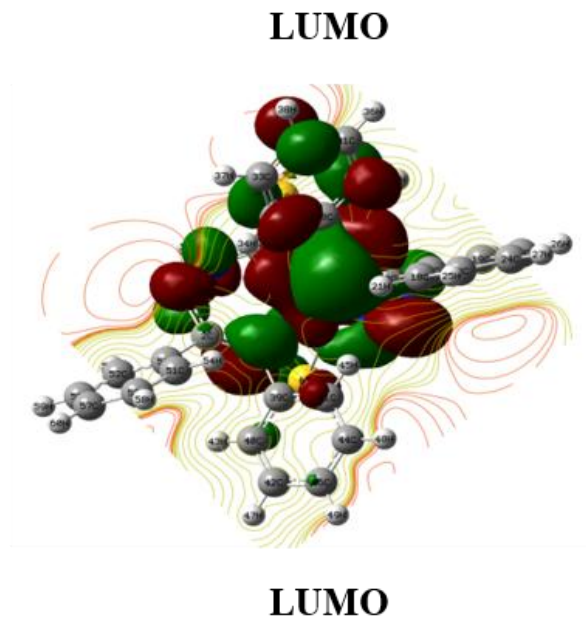

LUMO

(b)

Figure 9. The frontier molecular orbital density distributions of inhibitors (a) TMCD (b) TMPD.

In TMCD, the HOMO is delocalized strongly over the four nitrogen atoms, including two methyl substituents while the two nitrogen atom structure with two methyl groups is completely excluded. The HOMO in TMPD is totally localised over the nitrogen carbon backbone excluding the phenyl substituents. These results suggest that the nitrogen carbon backbone of the inhibitor has a greater tendency to donate electrons while the phenyl and methyl substituents in the structure have lesser tendency to donate electrons even though being an electron donating group.

The electron donating capacity of a molecule is associated with $E_{\mathrm{HOMO}}$, however electron accepecting tendency is associated with $E_{\text {LUMO }}$ [33]. Table 5 reveals that $E_{\text {HOMO }}$ value of TMCD is larger than TMPD, which supports the more electron donating capacity of TMCD. Further lower $E_{\text {LUMO }}$ value of TMCD favours its higher electron accepting tendency. Thus, order of inhibition efficiency is TMCD > TMPD.

The orbital energy gap i.e. $\Delta E$, provides the informtion about the interaction capacity of an inhibitor, which means lower the $\Delta E$ value, greater would be the interaction of the inhibitor with the metal surface [34]. The lower value of $\Delta E$ in TMCD supports its higher interaction with aluminium surface and thus results in a higher inhibition efficiency. 
The number of electrons transferred $(\Delta N)$ is calculated and given in Table 6 . The value of $\Delta N<3.6$ indicates the electron donating tendency of inhibitor molecules to metal surface. Thus, more the electron donating ability of the inhibitor, higher would be its inhibition efficiency. The order of electron donating ability in the present study is as follows: TMCD (0.61) > TMPD (0.28). This result also supports that TMCD is a better inhibitor than TMPD.

Table 6. Calculated quantum chemical parameters of the inhibitors.

\begin{tabular}{ccc}
\hline Quantum chemical parameters & TMCD & TMPD \\
\hline Dipole moment $(\mathrm{D})$ & 8.37 & 5.83 \\
$E_{\text {HOMO }}(\mathrm{eV})$ & -6.43 & -8.68 \\
$E_{\mathrm{LUMO}}(\mathrm{eV})$ & -1.36 & -0.98 \\
$\Delta E(\mathrm{eV})$ & 5.07 & 7.70 \\
Ionization potential $(\mathrm{IP})(\mathrm{eV})$ & 6.43 & 8.68 \\
Electron affinity $(\mathrm{EA})(\mathrm{eV})$ & 1.36 & 0.98 \\
Electronegativity $(\chi)$ & 3.89 & 4.83 \\
Hardness $(\eta)$ & 2.53 & 3.85 \\
Softness $(\sigma)$ & 0.39 & 0.25 \\
Electrophilicity $(\omega)$ & 3.49 & 2.53 \\
Fraction of electron transferred $(\Delta N)$ & 0.61 & 0.28 \\
\hline
\end{tabular}

Fukui function calculation has been carried out for confirming the active sites. The site which has highest absolute values of $f^{+}$and $f^{-}$would undergo the nucleophilic and electrophilic attack respectively [35]. Table 6 reports the Mulliken atomic charges for the non-hydrogen atoms of the molecules.

The results show that nitrogen atoms have the highest negative charge, followed by the phenyl and methyl substituent carbon atoms. These atoms are therefore likely to take part in an electrophilic attack in which case they will readily donate electrons to the electrophilic species. When the electrophilic species is the metal surface, the donated electrons are accepted in the partially filled or vacant orbitals of the metal, which allows the molecules to be adsorbed on the metal surface. All $\mathrm{C}$ atoms directly attached to $\mathrm{N}$ and $\mathrm{S}$ atoms are electron deficient (i.e., they have positive charge) and therefore could be subjected to nucleophilic attack.

The calculated condensed Fukui functions are reported in Table 7. Comparing the atomic charge of the atoms with their Fukui functions, the number of atoms preferably for electrophilic attack were more for TMCD than for TMPD. Hence TMCD has more susceptible sites for adsorption on the aluminium surface. Thus from the above statement the inhibitory effects of the studied inhibitors can be given as follows: TMCD > TMPD. 


\section{Conclusion}

TMCD and TMPD are effective corrosion inhibitors for aluminium in $1 \mathrm{M} \mathrm{HCl}$ medium. Polarization studies confirmed that the inhibitors are of mixed type but predominantly cathodic and their inhibition efficiency increases with increase in inhibitor concentration. EIS study showed the presence of large capacitive and inductive loops at high and low frequencies. Reactivity sites for nucleophilic and electrophilic attacks were indicated using the Fukui function.

\section{Acknowledgement}

Authors gratefully acknowledge the Ministry of Human Resource Development (MHRD), New Delhi, India for the financial assistance and facilitation of our study.

\section{References}

1. R. Rosliza, W.B. Wan Nik, S. Izman and Y. Prawoto, Curr. Appl. Phys., 2010, 10, 923.

2. A. Singh, Y. Lin, W. Liu, S. Yu, J. Pan, C. Ren and D. Kuanhai, J. Ind. Eng. Chem., 2014, 20, 4276.

3. I. Merimi, Y. El Ouadi, K.R. Ansari, H. Oudda, B. Hammouti, M.A. Quraishi, F.F. Alblewi, N. Rezki, M.R. Aouad and M. Messali, Anal. Bioanal. Electrochem., 2017, 9, 640.

4. A. Anejjar, R. Salghi, A. Zarrouk, O. Benali, H. Zarrok, B. Hammouti and E.E. Ebenso, J. Assoc. Arab Univ. Basic Appl. Sci., 2014, 15, 21.

5. K.R. Ansari and M.A. Quraishi, Anal. Bioanal. Electrochem., 2015, 7, 509.

6. R. Karthikaiselvi and S. Subhashini, J. Assoc. Arab Univ. Basic Appl. Sci., 2014, 16, 74.

7. I.B. Obot, N.O. Obi-Egbedi and S.A. Umoren, Corros. Sci., 2009, 51, 1868.

8. X. Li., S. Deng and H. Fu, Corros Sci., 2011, 53, 1529.

9. A. Yurt and O. Aykin, Corros Sci., 2011, 53, 3725.

10. Q. Zhang and Y. Hua, Mater. Chem. Phys., 2010, 119, 57.

11. V. Branzoi, F. Golgovici and F. Branzoi, Mater. Chem. Phys., 2010, 78, 122.

12. N.O. Eddy, H.M. Yahaya and E.E. Oguzie, J. Adv. Res., 2011, 6, 203.

13. D.P. Singh and R. Kumar, Trans. Met. Chem., 2006, 31, 970.

14. I.B. Obot, S.A. Umoren, Z.M. Gasem, R. Suleiman and B.E. Ali, J. Ind. Eng. Chem., 2015, 21, 1328.

15. U.K. Pandey, O.P. Pandeya, S.K. Sengupta and S.C. Tripathi, Polyhedron, 1987, 6, 1611.

16. M.J. Frisch et al., 2007, Gaussian 03, Revision E.01, Gaussian Inc., Wallingford CT.

17. X.H. Li, S.D. Deng and H. Fu, Corros. Sci., 2011, 53, 1529.

18. K.R. Ansari and M. A. Quraishi, J. Ind. Eng. Chem., 2014, 20, 2819.

19. K.R. Ansari and M. A. Quraishi, J. Assoc. Arab Univ. Basic Appl. Sci., 2015, 18, 12.

20. K.R. Ansari and M. A. Quraishi, Physica E, 2015, 69, 322. 
21. K.R. Ansari, M. A. Quraishi and A. Singh, Corros. Sci., 2014, 79, 5.

22. K.R. Ansari, M. A. Quraishi and A. Singh, J. Ind. Eng. Chem., 2015, 25, 89.

23. K.R. Ansari, Sudheer, A. Singh and M.A. Quraishi, J. Dispersion. Sci. Technol., 2015, 36, 908.

24. K.F. Khaled, A.S. Fadl-Allah and B. Hammouti, Mat. Chem. Phys., 2009, 117, 148.

25. X.H. Li and S.D. Deng, Corros. Sci., 2012, 65, 299.

26. M.A. Amin, Q. Mohsen and O.A. Hazzai, Mater. Chem. Phys., 2009, 114, 908.

27. H.J.W. Lenderink, M.V.D. Linden and J.H.W. De Wit, Electrochim. Acta, 1993, 38, 1989.

28. E.A. Noor, Mater. Chem. Phys., 2009, 114, 533.

29. T. Valand and K.E. Heusler, J. Electroanal. Chem., 1983, 149, 71.

30. S.D. Deng and X.H. Li, Corros. Sci., 2012, 64, 253.

31. M. Lagrenée, B. Mernari, M. Bouanis, M. Traisnel and F. Bentiss, Corros. Sci., 2002, 44, 573.

32. S.S. Abd El Rehim, H.H. Hassan and M.A. Amin, Mater. Chem. Phys., 2001, 70, 64.

33. I.B. Obot, D.D. Macdonald and Z.M. Gasem, Corros Sci., 2015, 99, 1.

34. I.B. Obot and Z.M. Gasem, Corros. Sci., 2014, 83, 356.

35. F. Jia-jin, L. Su-ning, W. Ying, C. Lin-hua and L. Lu-de, J. Mater. Sci., 2010, 45, 6255. 